Supporting Information

for

\title{
Controlled Demolition and Reconstruction of Imidazolate and Carboxylate Metal-Organic Frameworks by Acid Gas Exposure and Linker Treatment
}

Arvind Ganesan, ${ }^{\mathrm{a}}$ Stephen C. Purdy, ${ }^{\mathrm{b}}$ Zhenzi Yu, ${ }^{\mathrm{a}}$ Souryadeep Bhattacharyya, ${ }^{\mathrm{a}}$ Katharine Page, ${ }^{\mathrm{b}, \mathrm{c}}$ David S. Sholl, ${ }^{a}$ and Sankar Nair ${ }^{a *}$

${ }^{\text {aS }}$ School of Chemical \& Biomolecular Engineering, Georgia Institute of Technology, Atlanta, GA 30332, USA

bNeutron Scattering Division, Oak Ridge National Laboratory, Oak Ridge, TN 37831, USA

${ }^{\mathrm{c}}$ Materials Science and Engineering Department, University of Tennessee, Knoxville, TN 37996, USA

*Corresponding author: sankar.nair@chbe.gatech.edu

Keywords: MOFs, acid gas, reconstruction, healing 


\section{Supporting Tables}

Table S1. MOF exposure protocols leading to a significant loss in crystallinity and porosity.

\begin{tabular}{|c|c|}
\hline MOF & Acid gas exposure \\
\hline ZIF-8 & Dry $\mathrm{NO}_{2} 125 \mathrm{ppm}$-days (concentrationxtime) \\
\hline ZIF-8 & $\mathrm{Humid}_{(75 \% \mathrm{RH}) \mathrm{NO}_{2} 225 \mathrm{ppm} \text {-days }}$ \\
\hline ZIF-90 & Humid $(50 \% \mathrm{RH}) \mathrm{SO}_{2} 300$ ppm-days \\
\hline ZIF-71 & Dry $\mathrm{NO}_{2} 750 \mathrm{ppm}$-days \\
\hline UiO-66 & Dry $\mathrm{NO}_{2} 759$ ppm-days \\
\hline UiO-67 & Humid $(75 \% \mathrm{RH}) \mathrm{SO}_{2} 100$ ppm-days \\
\hline
\end{tabular}

Table S2. SACRed recovery protocol for each MOF-acid gas pair.

\begin{tabular}{|c|c|c|}
\hline MOF & Acid gas & SACRed Protocol \\
\hline ZIF-8 & Dry $\mathrm{NO}_{2}$ & 2-methylimidazole in Methanol, $90{ }^{\circ} \mathrm{C}$ \\
\hline ZIF-8 & ${\text { Humid } \mathrm{NO}_{2}}$ & 2-methylimidazole in Methanol, $90{ }^{\circ} \mathrm{C}$ \\
\hline ZIF-90 & ${\text { Humid } \mathrm{SO}_{2}}^{\circ}$ & 2-Imidazolecarboxaldehyde in Methanol, $90^{\circ} \mathrm{C}$ \\
\hline ZIF-71 & Dry $\mathrm{NO}_{2}$ & 4,5- dichloro imidazole in Methanol, $90{ }^{\circ} \mathrm{C}$ \\
\hline UiO-66 & Dry $\mathrm{NO}_{2}$ & 1,4- benzene dicarboxylic acid in DMF, $90{ }^{\circ} \mathrm{C}$ \\
\hline UiO-67 & Humid $\mathrm{NO}_{2}$ & Biphenyl-4,4'-dicarboxylic acid in DMF, $90^{\circ} \mathrm{C}$ \\
\hline
\end{tabular}


Table S3. Morphological characteristics of MOFs (pristine, acid gas exposed and SACRed treated), *ZIF-8 SACRed-treated after humid $\mathrm{SO}_{2}$ exposure shows complete recovery in surface area and pore volume ${ }^{13} . * *$ BET surface areas are calculated using both the Rouquerol criteria and the constant $P / P_{0}$ range $(0-0.3)$ method. ${ }^{\#} 100 \%$ recovery denotes surface area or pore volume equal to or more than the pristine value.

\begin{tabular}{|c|c|c|c|c|c|}
\hline MOF & Description & $\begin{array}{l}\text { BET Surface } \\
\text { area }\left(\mathrm{m}^{2} / \mathrm{g}\right)^{* *} \\
\text { Rouquerol// } \\
\text { Constant } \\
P / P_{0}\end{array}$ & $\begin{array}{c}\text { Pore } \\
\text { volume } \\
\left(\mathrm{cm}^{3} / \mathbf{g}\right)\end{array}$ & $\begin{array}{l}\text { Surface } \\
\text { Area \% }\end{array}$ & $\begin{array}{c}\text { Pore } \\
\text { Volume \% }\end{array}$ \\
\hline \multirow{3}{*}{ ZIF-8* } & Pristine & $1651 / 1250$ & 0.61 & $100 / 100$ & 100 \\
\hline & Dry $\mathrm{NO}_{2} 125$ ppm-days & $793 / 665$ & 0.36 & $48 / 59$ & 59 \\
\hline & Dry $\mathrm{NO}_{2} \mathrm{SACRed}$ & $1494 / 1153$ & 0.53 & $90 / 87$ & 87 \\
\hline \multirow{3}{*}{ ZIF-8 } & Pristine & $1651 / 1250$ & 0.61 & $100 / 100$ & 100 \\
\hline & Humid $\mathrm{NO}_{2} 225$ ppm-days & $560 / 460$ & 0.23 & $34 / 37$ & 38 \\
\hline & Humid $\mathrm{NO}_{2}$ SACRed & $1261 / 985$ & 0.49 & $76 / 80$ & 80 \\
\hline \multirow{3}{*}{ ZIF-90 } & Pristine & $1329 / 949$ & 0.42 & $100 / 100$ & 100 \\
\hline & Humid $\mathrm{SO}_{2} 300$ ppm-days & $387 / 297$ & 0.12 & $29 / 28$ & 29 \\
\hline & Humid $\mathrm{SO}_{2}$ SACRed & $945 / 787$ & 0.34 & $71 / 76$ & 81 \\
\hline \multirow{3}{*}{ ZIF-71 } & Pristine & $994 / 792$ & 0.35 & $100 / 100$ & 100 \\
\hline & Dry $\mathrm{NO}_{2} 750$ ppm-days & $623 / 490$ & 0.22 & $63 / 63$ & 63 \\
\hline & Dry $\mathrm{NO}_{2}$ SACRed & $803 / 665$ & 0.29 & $81 / 83$ & 83 \\
\hline \multirow{3}{*}{ UiO-66 } & Pristine & $1170 / 911$ & 0.43 & $100 / 100$ & 93 \\
\hline & Dry $\mathrm{NO}_{2} 750$ ppm-days & $857 / 627$ & 0.35 & $73 / 81$ & 76 \\
\hline & Dry $\mathrm{NO}_{2}$ SACRed & $1060 / 736$ & 0.4 & $91 / 93$ & 87 \\
\hline \multirow{3}{*}{ UiO-67 } & Pristine & $1854 / 1540$ & 0.65 & $100 / 100$ & 100 \\
\hline & Humid $\mathrm{NO}_{2} 100$ ppm-days & $0 / 0$ & 0 & $0 / 0$ & 0 \\
\hline & Humid $\mathrm{NO}_{2}$ SACRed & $1991 / 1666$ & 0.66 & $100 / 100^{\#}$ & $100^{\#}$ \\
\hline
\end{tabular}


Table S4. Calculated surface area of ZIF-8 with $50 \%$ and $100 \%$ functionalization of the 2methylimidazole linker in the 4-carbon position. The computationally derived structures are available in the accompanying zip file.

\begin{tabular}{|c|c|}
\hline MOF & Surface $\operatorname{area}\left(\mathbf{m}^{\mathbf{2}} \mathbf{g}\right)$ \\
\hline ZIF-8 & 1387.8 \\
\hline ZIF-8 (50\% organic nitro functionalization) & 991.5 \\
\hline ZIF-8 (100\% organic nitro functionalization) & 742.7 \\
\hline
\end{tabular}

Table S5. Summary of functional groups attributed to new FTIR peaks in MOFs exposed to acid gases.

\begin{tabular}{|c|c|c|c|c|c|c|}
\hline $\begin{array}{c}\text { Functional } \\
\text { Group }\end{array}$ & $\begin{array}{c}\text { ZIF-8 } \\
\text { Dry NO2 }\end{array}$ & $\begin{array}{c}\text { ZIF-8 } \\
\text { Humid } \\
\mathrm{NO}_{2}\end{array}$ & $\begin{array}{c}\text { ZIF-71 } \\
\text { Dry NO2 }\end{array}$ & $\begin{array}{c}\text { UiO-66 } \\
\text { Dry NO2 }\end{array}$ & $\begin{array}{c}\text { UiO-67 } \\
\text { Humid } \\
\mathrm{NO}_{2}\end{array}$ & Expected Range \\
\hline \multicolumn{7}{|c|}{ Organic Groups } \\
\hline \multirow{6}{*}{ Nitro } & & $\begin{array}{l}1500- \\
1540\end{array}$ & 1540 & 1550 & 1520 & $\begin{array}{l}1485-1570 \text { (asym. } \\
\mathrm{NO}_{2} \text { str.) }\end{array}$ \\
\hline & & & & & & $\begin{array}{l}1320-1370 \text { (sym. } \\
\mathrm{NO}_{2} \text { str.) }\end{array}$ \\
\hline & & 1031 & 1020 & 1100 & 1100 & $\begin{array}{l}865-1180 \\
\text { (aromatic C-N str.) }\end{array}$ \\
\hline & & & & & & $\begin{array}{l}850-1000 \\
\text { (aliphatic C-N str.) }\end{array}$ \\
\hline & & & 840 & & & $\begin{array}{l}830-865\left(\mathrm{NO}_{2}\right. \\
\text { def.) }\end{array}$ \\
\hline & & & & & & $\begin{array}{l}605-670\left(\mathrm{NO}_{2} \text { def. }\right. \\
\text { aliphatic) }\end{array}$ \\
\hline \multirow{4}{*}{ Nitrite } & & 1650 & 1660 & 1660 & 1660 & $\begin{array}{l}1610-80(\mathrm{~N}=\mathrm{O} \\
\text { str. })\end{array}$ \\
\hline & & 812 & 810 & 780 & 780 & $750-850$ (N-O str.) \\
\hline & & 674 & 885 & 1105 & 1105 & $\begin{array}{l}615-690(\mathrm{O}-\mathrm{N}=\mathrm{O} \\
\text { def. }\end{array}$ \\
\hline & & 1105 & 1150 & & & $1000-1300(\mathrm{C}-\mathrm{O})$ \\
\hline
\end{tabular}




\begin{tabular}{|c|c|c|c|c|c|c|}
\hline \multirow{6}{*}{ Nitrate } & 1270 & & & & 1655 & $\begin{array}{l}\text { 1615-1660 (asym. } \\
\mathrm{NO}_{2} \text { str.) }\end{array}$ \\
\hline & & & & & 1270 & $\begin{array}{l}\text { 1270-1300 (sym. } \\
\mathrm{NO}_{2} \text { str.) }\end{array}$ \\
\hline & & & & & & $840-870$ (N-O str.) \\
\hline & & & & & & $\begin{array}{l}\text { 745-765 }\left(\mathrm{NO}_{2}\right. \\
\text { def.) }\end{array}$ \\
\hline & & & & & & $\begin{array}{l}680-720\left(\mathrm{NO}_{2}\right. \\
\text { def. })\end{array}$ \\
\hline & & & & & & $1000-1300(\mathrm{C}-\mathrm{O})$ \\
\hline \multirow{4}{*}{ Nitramine } & & 1570 & 1560 & & & $\begin{array}{l}\text { 1530-1630 (asym. } \\
\mathrm{NO}_{2} \text { str.) }\end{array}$ \\
\hline & & & 1295 & & & $\begin{array}{l}\text { 1260-1315 (sym. } \\
\mathrm{NO}_{2} \text { str.) }\end{array}$ \\
\hline & & 970 & 995 & & & $\begin{array}{l}\text { 925-1030 (N-N } \\
\text { str.) }\end{array}$ \\
\hline & & 1500 & 790 & & & $\begin{array}{l}755-775\left(\mathrm{NO}_{2}\right. \\
\text { def. })\end{array}$ \\
\hline \multirow{3}{*}{ Nitrosamine } & & & 1495 & & & $\begin{array}{l}1450-1500(\mathrm{~N}=\mathrm{O} \\
\text { str.) }\end{array}$ \\
\hline & & 970 & & & & $\begin{array}{l}925-1030(\mathrm{~N}-\mathrm{N} \\
\text { str.) }\end{array}$ \\
\hline & & 1165 & 1190 & & & $\begin{array}{l}1160-1200(\mathrm{C}-\mathrm{N} \\
\text { str.) }\end{array}$ \\
\hline \multicolumn{7}{|c|}{ Inorganic Groups } \\
\hline \multirow{3}{*}{ Nitrate } & & 1380 & 1385 & 1360 & 1360 & $\begin{array}{l}\text { 1350-1410 (asym. } \\
\mathrm{NO}_{3} \text { str.) }\end{array}$ \\
\hline & & 860 & 840 & 850 & 850 & $\sim 800-860$ \\
\hline & & 712 & & 720 & 720 & $\sim 710-730$ \\
\hline \multirow{2}{*}{ Nitrite } & 1235 & 1235 & & & & $\begin{array}{l}\text { 1235-1275 (asym. } \\
\mathrm{NO}_{2} \text { str.) }\end{array}$ \\
\hline & 812 & 812 & & & & $800-835$ \\
\hline
\end{tabular}




\section{Supporting Figures}
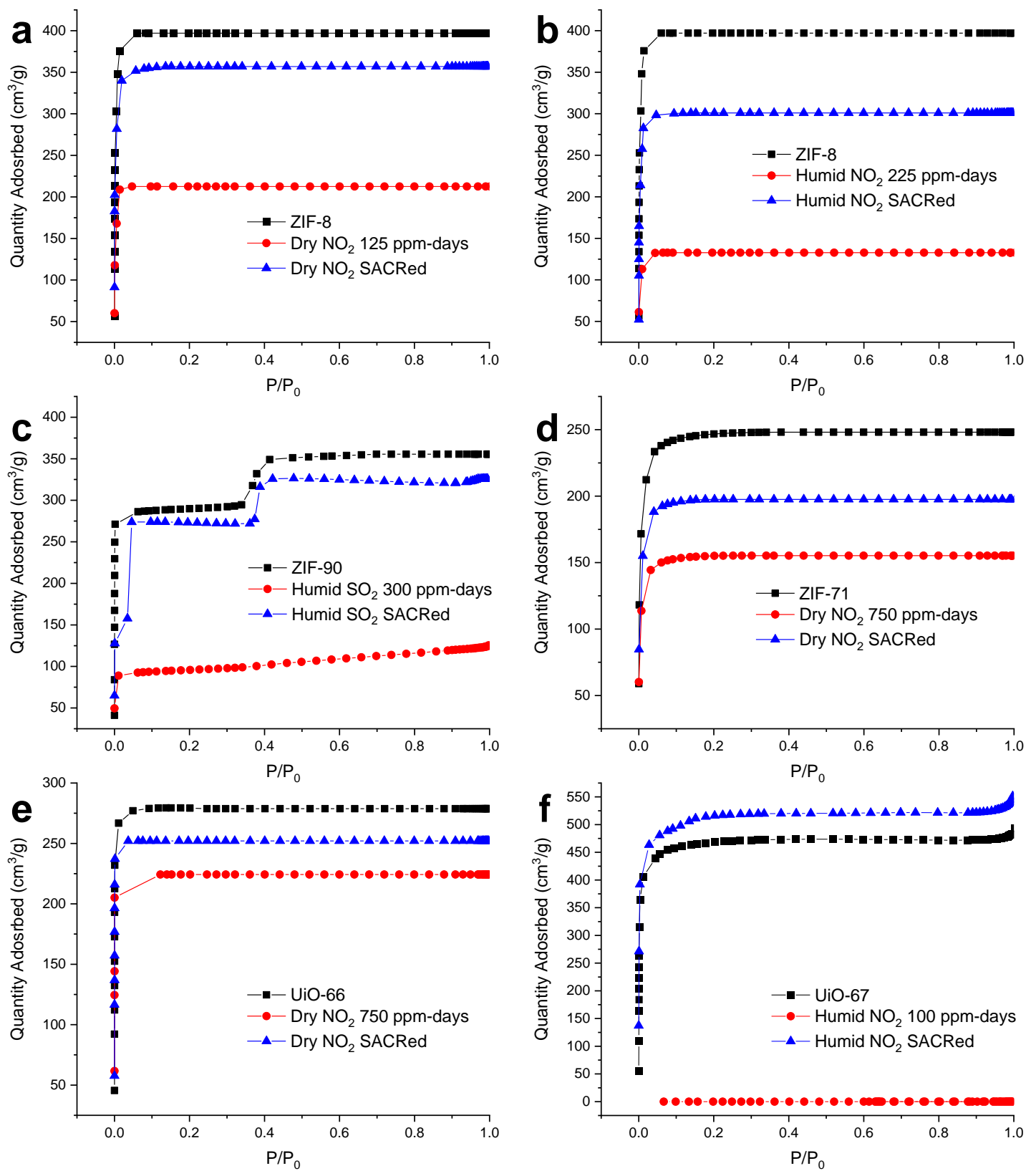

Figure S1. $\mathrm{N}_{2}$ adsorption isotherms of (a) ZIF-8 (pristine, dry $\mathrm{NO}_{2}$ exposed 125 ppm-days and post SACRed treatment), (b) ZIF-8 (pristine, humid $\mathrm{NO}_{2}$ exposed 225 ppm-days and post SACRed treatment), (c) ZIF-90 (pristine, humid $\mathrm{SO}_{2}$ exposed 300 ppm-days and post SACRed treatment), (d) ZIF-71 (pristine, dry $\mathrm{NO}_{2}$ exposed 750 ppm-days and post SACRed treatment), (e) UiO-66 (pristine, dry $\mathrm{NO}_{2}$ exposed 750 ppm-days and post SACRed treatment) and (f) UiO-67 (pristine, humid $\mathrm{NO}_{2}$ exposed to 100 ppm-days and post SACRed treatment). 

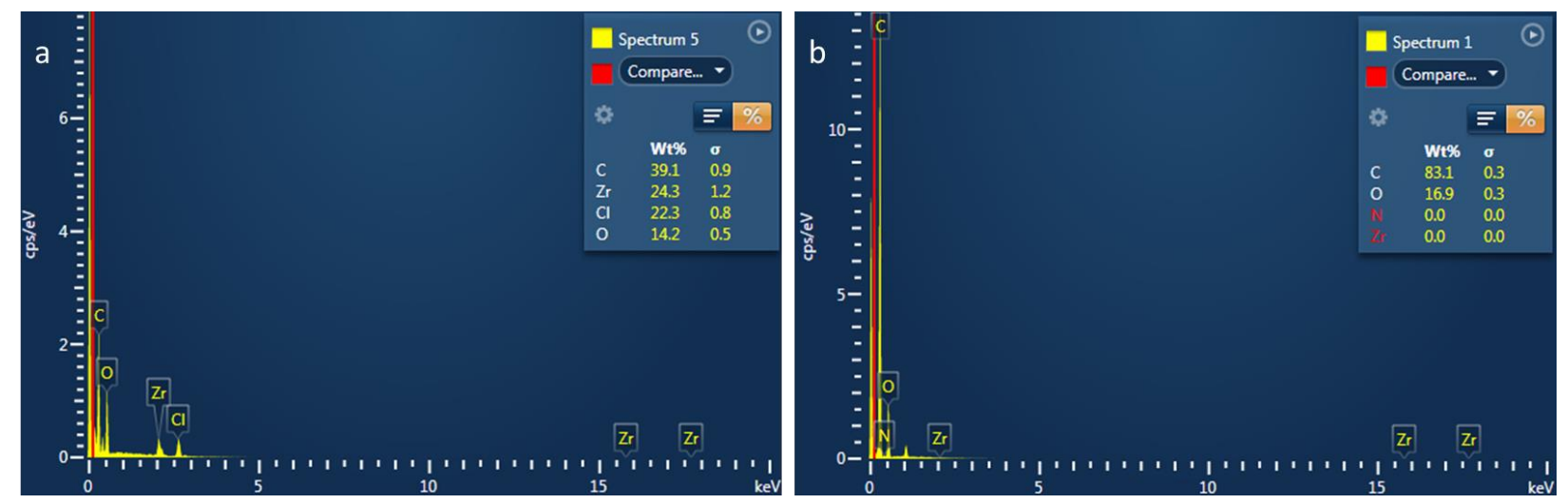

Figure S2. EDX measurements (a) UiO-67 synthesis solution and (b) UiO-67 SACRed recovery solution dried over conductive carbon tape.

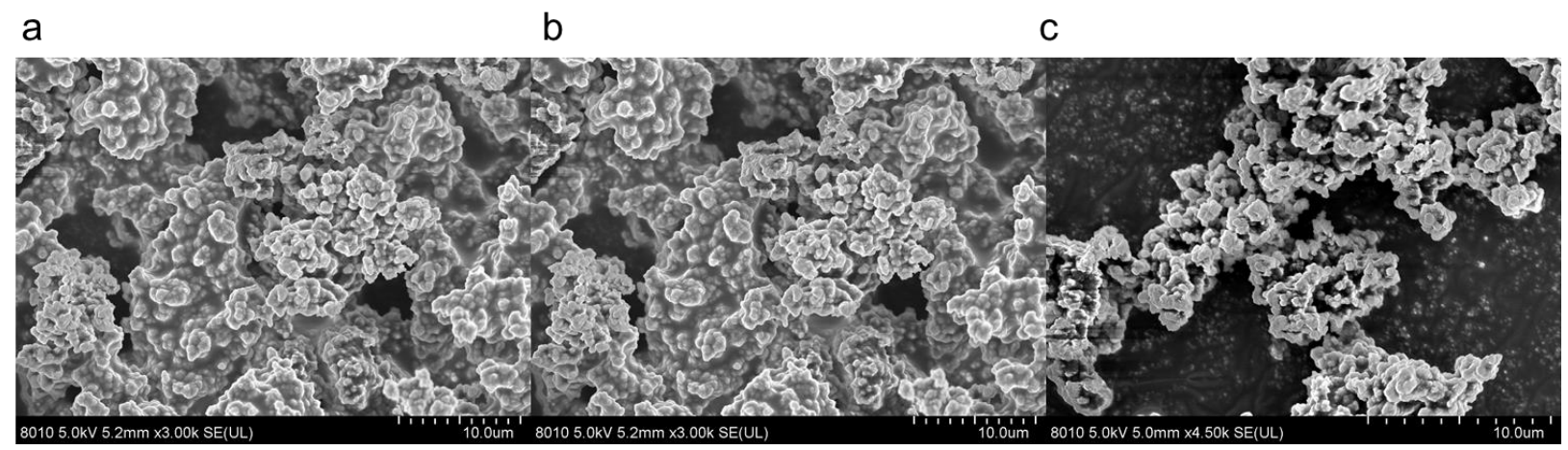

Figure S3. SEM images of (a) UiO-66, (b) UiO-66 Dry $\mathrm{NO}_{2} 750$ ppm-days and (c) UiO-66 Dry $\mathrm{NO}_{2}$ SACRed. The scale bar on all the images is $10 \mu \mathrm{m}$. 